\title{
molecules
}

ISSN 1420-3049

www.mdpi.org/molecules

Article

\section{Oligomerization of Indole Derivatives with Incorporation of Thiols}

\author{
Felikss Mutulis ${ }^{1,2}$, Adolf Gogoll ${ }^{3}$, Ilze Mutule ${ }^{1,2}$, Sviatlana Yahorava ${ }^{1}$, Aleh Yahorau ${ }^{1}$, \\ Edvards Liepinsh ${ }^{2}$ and Jarl E.S. Wikberg ${ }^{1}$
}

${ }^{1}$ Department of Pharmaceutical Biosciences, Division of Pharmaceutical Pharmacology, Uppsala Biomedical center, Uppsala University, S-751 24, Uppsala, Sweden; E-mails:

Sviatlana.Yahorava@farmbio.uu.se; Aleh.Yahorau@farmbio.uu.se; Jarl.Wikberg@farmbio.uu.se

${ }^{2}$ Latvian Institute of Organic Synthesis, Aizkraukles 21, LV-1006, Riga, Latvia; E-mail: ilzem@osi.lv; edv@osi.lv

${ }^{3}$ Department of Biochemistry and Organic Chemistry, Box 576, Uppsala University, S-751 23, Uppsala, Sweden; E-mail: Adolf.Gogoll@biorg.uu.se

* Author to whom correspondence should be addressed. E-mail: fmutulis@osi.lv.

Received: 10 June 2008; in revised form: 18 August 2008 / Accepted: 19 August 2008 / Published: 26 August 2008

\begin{abstract}
Two molecules of indole derivative, e.g. indole-5-carboxylic acid, reacted with one molecule of thiol, e.g. 1,2-ethanedithiol, in the presence of trifluoroacetic acid to yield adducts such as 3-[2-(2-amino-5-carboxyphenyl)-1-(2-mercaptoethylthio)ethyl]-1Hindole-5-carboxylic acid. Parallel formation of dimers, such as 2,3-dihydro-1H,1'H-2,3'biindole-5,5'-dicarboxylic acid and trimers, such as 3,3'-[2-(2-amino-5-carboxyphenyl)ethane-1,1-diyl]bis(1H-indole-5-carboxylic acid) of the indole derivatives was also observed. Reaction of a mixture of indole and indole-5-carboxylic acid with 2phenylethanethiol proceeded in a regioselective way, affording 3-[2-(2-aminophenyl)-1(phenethylthio)ethyl]-1H-indole-5-carboxylic acid. An additional product of this reaction was 3-[2-(2-aminophenyl)-1-(phenethylthio)ethyl]-2,3-dihydro- $1 \mathrm{H}, 1$ ' $H$-2,3'-biindole-5'carboxylic acid, which upon standing in DMSO-d $\mathrm{d}_{6}$ solution gave 3-[2-(2-aminophenyl)-1(phenethylthio)ethyl]-1H,1'H-2,3'-biindole-5'-carboxylic acid. Structures of all compounds were elucidated by NMR, and a mechanism for their formation was suggested.
\end{abstract}


Keywords: Side reaction, oligomerization of indole derivatives, incorporation of thiols, regioselectivity, NMR study.

\section{Introduction}

Multistage solid phase organic synthesis, which excludes isolation of intermediates steps, is an attractive method for the preparation of diverse chemical substances [1]. However, a high yield of desired product at each synthetic stage is particularly important for these methods [2], as various side reactions, lowering the chemical yield of the individual synthetic steps, are the main limitation of the method [3]. In order to make the method more efficient, a deeper understanding of these unwanted processes is desirable.

Scheme 1. Incorporation of thiols. Proposed mechanism and related substances.<smiles></smiles>

$1,1 \mathrm{a}$
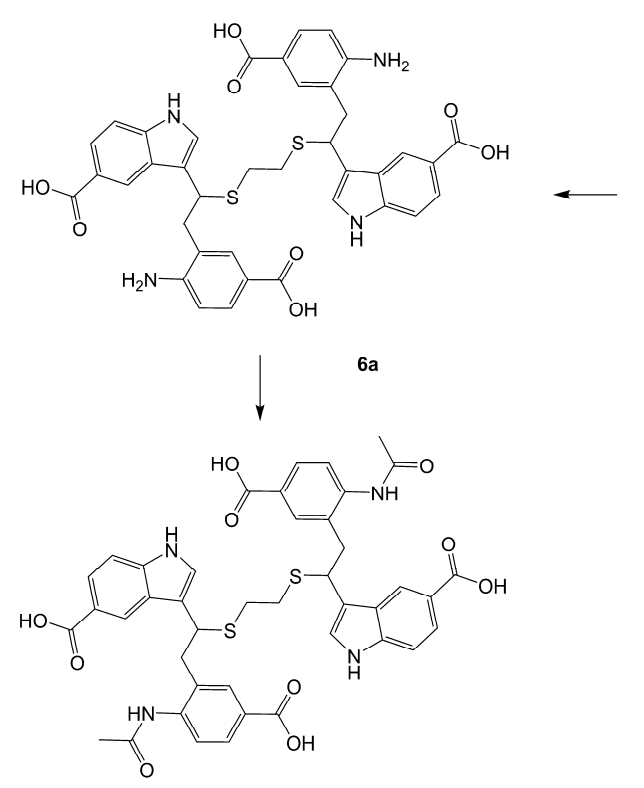

6b

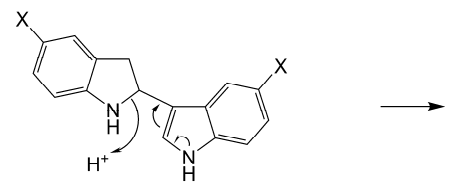

2, 2a
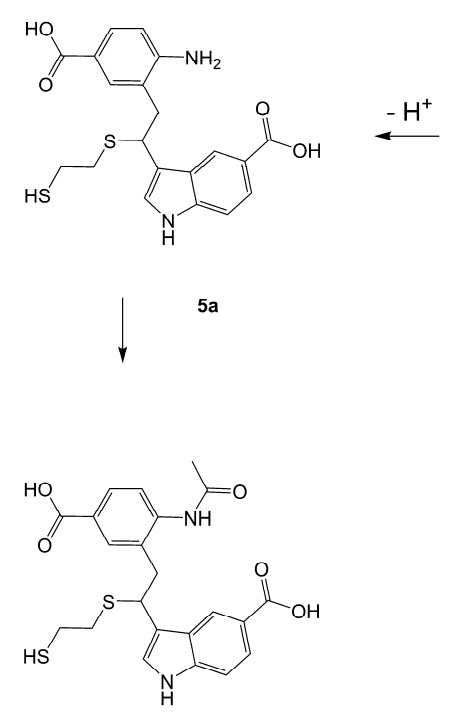

5b

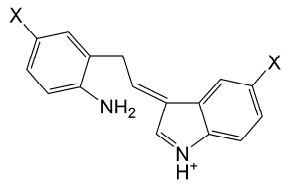

$4,4 a$<smiles>Nc1ccc(C(=O)O)cc1CCCCCc1cc2ccc(C(=O)O)cc2[nH]1</smiles>

4a

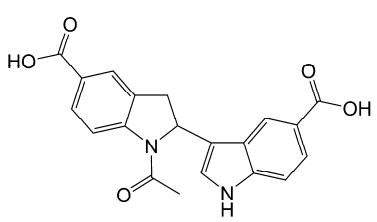

$2 b$

$$
\begin{aligned}
& \text { 1, 2, } 4-X=H \\
& \text { 1a, 2a, 4a- } X=\mathrm{COOH}
\end{aligned}
$$

In our previous investigation of indole-5-carboxylic acid derivatives attached to carboxylated Wang polymer [4], LC/MS analysis after the cleavage step using trifluoroacetic acid and 1,2-ethanedithiol mixture unexpectedly showed the presence of compounds whose molecular mass corresponded to dimerization of the expected products with addition of 1,2-ethanedithiol. 
Dimers [5-10], trimers [8, 11-17] and tetramers [7, 14, 15, 17] of indole and its derivatives are described in a number of research articles and patents. It is well documented that indole (1) forms the indole dimer 2,3-dihydro-1H,1'H-2,3'-biindole $[6,15]$ (2, Scheme 1) and trimer 2-[2,2-di(1H-indol-3yl)ethyl]-aniline $[5,12,15]$ (3, Figure 1$)$ under various acidic conditions.

\section{Results and Discussion}

To investigate the aforementioned unknown side reaction, in the present study we tried to prepare a similar compound from indole-5-carboxylic acid itself. Indole-5-carboxylic acid (1a, Scheme 1) was treated with trifluoroacetic acid and 1,2-ethanedithiol mixture. Indeed, the expected dimerization and addition reactions furnished the desired product. In addition, according to LC/MS, along with the dimer and trimer of indole-5-carboxylic acid, a product, whose molecular mass corresponded to the sum of mass of four molecules of indole-5-carboxylic acid and one molecule 1,2-ethanedithiol was detected.

Figure 1. Indole trimers.

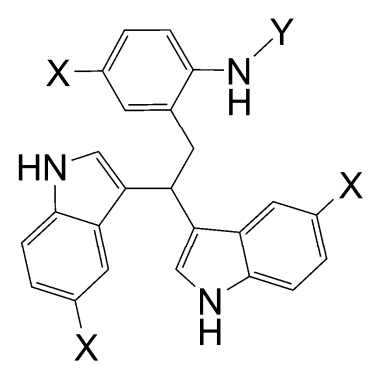

$$
\begin{aligned}
& \mathbf{3}(X=Y=H) \\
& \text { 3a }(X=\mathrm{COOH}, Y=H) \\
& \text { 3b }\left(X=\mathrm{COOH}, Y=\mathrm{COCH}_{3}\right) \\
& \text { 3c }(X=\mathrm{Cl}, Y=\mathrm{H})
\end{aligned}
$$

A detailed NMR investigation of the products was performed using ${ }^{1} \mathrm{H}$ [18], COSY [18, 19], proton decoupled ${ }^{13} \mathrm{C}$ [18, 20], HETRES [21] and selective long range INEPT [22] experiments, and evaluation of the resulting NMR data confirmed proposed structures 2a (Scheme 1), 3a (Figure 1), 5a and 6a (Scheme 1). Moreover, as additional proof of the correctness of the proposed structures, their corresponding acetylated derivatives $\mathbf{2 b}$ (Scheme 1), $\mathbf{3 b}$ (Figure 1), $\mathbf{5 b}$ and $\mathbf{6 b}$ (Scheme 1) were also prepared and their structures investigated.

1,2-Ethanedithiol is known to be a strong nucleophilic reagent, widely used as the most effective scavenger of carbonium ions [23]. Incorporation of dithiol could be explained in terms of the mechanism proposed by Smith [11] and Sundberg [24] for oligomerization of indole (Scheme 1). We suggest that ethanedithiol reacts with the indole-5-carboxylic acid dimer 4a (Scheme 1) forming an ethanedithiol adduct 5a. Competitive attachment of the third molecule of indole-5-carboxylic acid leads to parallel formation of trimer 3a (Figure 1). It is logical to assume that in a way similar to formation of $\mathbf{5 a}$, the $\mathrm{SH}$ group in $\mathbf{5 a}$ reacts further with the protonated dimer $\mathbf{4 a}$ yielding a symmetric structure 6a (Scheme 1).

Further, we investigated the influence of substitution pattern in the indole on the course of the oligomerization. Replacing 1,2-ethanedithiol with 2-phenylethanethiol and varying substituents in the indole system we obtained products 7a-f analogous to 5a (Scheme 2). Indole-5-carboxylic acid, its diethylamide [24], indole-6-carboxylic acid, 5-cyanoindole, 5-fluoroindole and 5-chloroindole were 
used. Acetylated derivatives of 2-phenylethanethiol adducts 8a-c (Scheme 2) were prepared to assist in the spectroscopic structure assignments. Incorporation of thiols was accompanied by formation of ample quantities of dimers of indoles like $\mathbf{2 a}$, acetylated derivative $\mathbf{2 b}$ (Scheme 1) and indole trimers like 3a, acetylated derivative $\mathbf{3 b}$, and $\mathbf{3 c}$ (Figure 1) (according to LC/MS data).

Scheme 2. Incorporation of 2-phenylethanethiol.

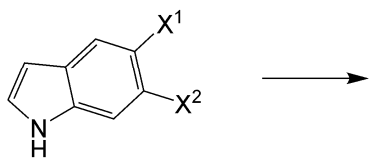

$1(a-f)$

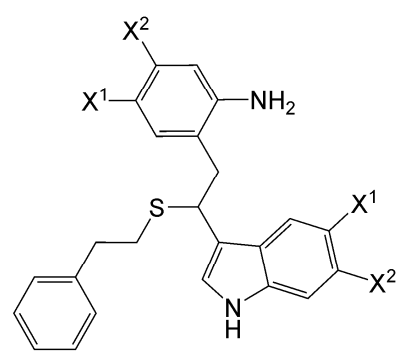

$7(a-f)$ 1a, 7a, 8a: $X^{1}=\mathrm{COOH}, X^{2}=\mathrm{H}$

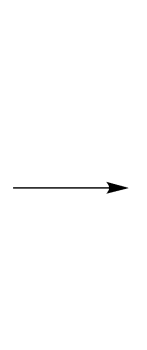

1d, 7d: $X^{1}=C N, X^{2}=H$

1b, 7b, 8b: $X^{1}=\mathrm{H}, \mathrm{X}^{2}=\mathrm{COOH}$

1c, 7c, 8c: $X^{1}=\operatorname{CON}\left(\mathrm{C}_{2} \mathrm{H}_{5}\right)_{2}, X^{2}=H$

1e, 7e: $X^{1}=F, X^{2}=H$

1f, 7f: $\quad X^{1}=\mathrm{Cl}, X^{2}=H$

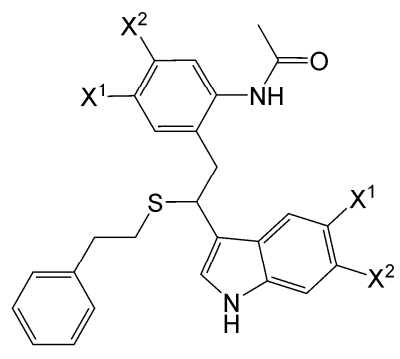

$8(a-c)$

It was not possible to obtain an adduct from unsubstituted indole and 1,2-ethanedithiol under typical reaction conditions. Incorporation of this indole was only possible when it was used in a mixture with indole-5-carboxylic acid. In addition, it was noted that the reaction proceeded in a regioselective way yielding 9 (Scheme 3). This reactivity pattern of unsubstituted indole could be explained on the basis of mechanism shown in Scheme 1. Michael additions of thiols are known to be facilitated by the electrodeficiency of the participating alkenes [26]. Electron acceptors attached to the benzene ring of indole should decrease the electron density on the carbon atom being attacked by thiols in structures like $\mathbf{4 a}$ (Scheme 1), thereby promoting the reaction.

Scheme 3. Introduction of unsubstituted indole.
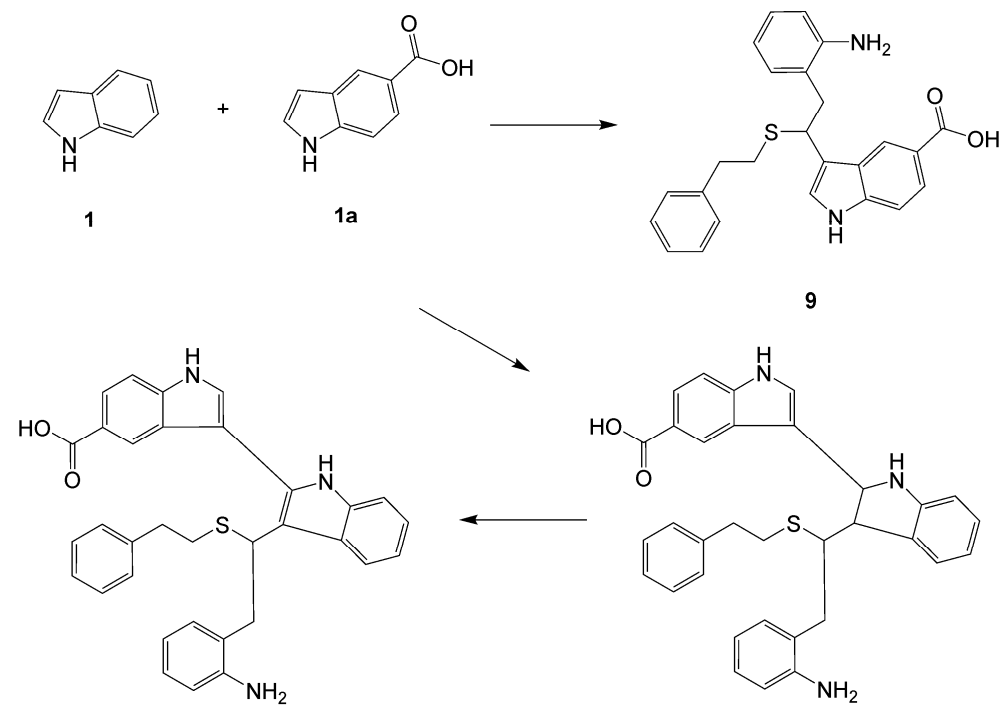

11 
An additional indole oligomerization product 10, corresponding to the combination of two molecules of indole, one molecule of indole-5-carboxylic acid and one molecule of 2-phenylethanethiol was isolated from the reaction mixture (Scheme 3). Upon standing in DMSO- $\mathrm{d}_{6}$ solution for one week, the indoline structure in $\mathbf{1 0}$ was oxidized quantitatively to yield indole $\mathbf{1 1 .}$

Yields for the thiol incorporation products reached 30\% (Table 1). They were higher if carbonyl (compounds 5a, 7a-c, 9) or nitrile (7d) groups were attached to the starting indole molecule. Much less reactive were halogenated indoles (products $7 \mathbf{e}$ and $\mathbf{7 f}$ ). Particular low were the yields for tetramer $\mathbf{6 a}$ and trimer $\mathbf{1 0 .}$

Table 1. Representative yields for products of incorporation of thiols.

\begin{tabular}{|l|cccccccccc|}
\hline Compound & $\mathbf{5 a}$ & $\mathbf{6 a}$ & $\mathbf{7 a}$ & $\mathbf{7 b}$ & $\mathbf{7 c}$ & $\mathbf{7 d}$ & $\mathbf{7 e}$ & $\mathbf{7 f}$ & $\mathbf{9}$ & \\
\hline Yield, \% & 33 & 1.3 & 19 & 32 & 30 & 15 & 2 & 5 & 19 & 1 \\
\hline
\end{tabular}

\section{Conclusions}

In summary, we have discovered a previously unknown indole oligomerization with incorporation of thiols, which has potential synthetic utility. The reaction proceeds with parallel formation of indole 3,3'-trimers; both processes require indole derivatives that are free of substitution at both the 2- and 3positions. The inclusion of electronegative substituents in benzene ring of indole is another crucial factor that makes incorporation of thiols possible. Adducts similar to those reported herein might be of interest as, e.g., potential enzyme inhibitors.

\section{Experimental}

\section{General}

Reagents were obtained from Aldrich or Fluka. Evaporations of solvents were carried out on a vacuum rotary evaporator at $30{ }^{\circ} \mathrm{C}$ and 20 mbar. TLC was performed using Merck Silica gel 60 F 254 glass plates; flash chromatography was performed using Merck Silica gel (70-230 mesh, pore size $60 \AA$ ). LC/MS was performed on a Perkin Elmer PE SCIEX API 150EX instrument with a Turboionspray Ion Source and equipped with a Dr. Maisch Reprosil-Pur C18-AQ, $5 \mu, 150 \times 3 \mathrm{~mm}$ HPLC column, using a water and acetonitrile gradient with $5 \mathrm{mM}$ ammonium acetate additive. Semipreparative HPLC was carried out on a LKB system consisting of a 2150 HPLC Pump, 2152 LC Controller and 2151 Variable Wavelength Monitor and Vydac RP $\mathrm{C}_{18}$ column $(10 \times 250 \mathrm{~mm}, 90 \AA$, $201 \mathrm{HS} 1010$ ), the eluent being an appropriate concentration of $\mathrm{MeCN}$ in water $+0.1 \% \mathrm{TFA}$, flow rate $5 \mathrm{~mL} / \mathrm{min}$, detection at $280 \mathrm{~nm}$. Freeze-drying was performed at 0.1 milibar on a Lyovac GT2 FreezeDryer (Finn-Aqua) equipped with a Busch 010-112 vacuum pump and a liquid nitrogen trap. Exact molecular masses were determined on a Micromass Q-Tof2 mass spectrometer equipped with an electrospray ion source. ${ }^{1} \mathrm{H}-\mathrm{NMR}$ spectra were recorded on a Jeol JNM-EX270 or Jeol JNM-EX400, Bruker DMX-600 spectrometer equipped with a cryoprobe or a Bruker DMX-500 spectrometer. 
Chemical shifts are reported in ppm relative to residual solvent signal $\left[\delta\left({ }^{1} \mathrm{H}\right) 2.50 \mathrm{ppm}, \delta\left({ }^{13} \mathrm{C}\right) 39.5\right.$ ppm]. Two-dimensional spectra recorded included COLOC, HETRES, selective long range INEPT, COSY, ROESY, sensitivity-enhanced ${ }^{13} \mathrm{C}$-HSQC and ${ }^{13} \mathrm{C}-{ }^{1} \mathrm{H}$ HMBC. ROESY mixing time was $0.1 \mathrm{~s}$. Pulsed-field gradients were used for all ${ }^{13} \mathrm{C}$ correlation spectra. ${ }^{13} \mathrm{C}$-HMBC spectra were recorded with coupling evolution delay for the generation of multiple-bond correlations set to $62.5 \mathrm{~ms}$. ROESY, ${ }^{13} \mathrm{C}$ HSQC and ${ }^{13} \mathrm{C}-{ }^{1} \mathrm{H}$ HMBC spectra were run with $4096 * 1024$ points data matrix, giving $\tau_{2 \max }=250 \mathrm{~ms}$ for ${ }^{1} \mathrm{H}$ nucleus in acquisition dimension and $\tau_{1 \max }=200 \mathrm{~ms}$ for ${ }^{1} \mathrm{H}$ or $\tau_{1 \max }=50 \mathrm{~ms}$ for ${ }^{13} \mathrm{C}$ for indirect dimension; prior to Fourier transform the data matrix was zero-filled twice and multiplication by shifted sine-bell window function applied. For ${ }^{1} \mathrm{H}-{ }^{13} \mathrm{C} \mathrm{HMBC}$ the magnitude spectra were calculated.

\section{Procedure A: Preparation of compounds 2a, 3a, 5a and 6a}

Indole-5-carboxylic acid (1a, $370 \mathrm{mg}, 2.3 \mathrm{mmol})$ was dissolved in a mixture of trifluoroacetic acid $(5 \mathrm{~mL})$ and 1,2-ethanedithiol $(1 \mathrm{~mL})$. After $1 \mathrm{~h}$ at room temperature the mixture was evaporated without heating under water aspirator pump vacuum. Dry ether-hexane $(1: 1,50 \mathrm{~mL})$ was added to the residue and the white precipitate formed was filtered off, washed with 1:1 dry ether-hexane and dried in vacuo. The raw product was next dissolved in $24 \% \mathrm{MeCN}$ in water, centrifuged and the clear solution applied in several portions onto an HPLC semipreparative column $(10$ x $250 \mathrm{~mm}$, Vydac RP $\mathrm{C}_{18} 90 \AA$ Pharmaceutical 201HS1010), eluent $-20 \% \mathrm{MeCN}$ in water $+0.1 \% \mathrm{TFA}$, flow $5 \mathrm{~mL} / \mathrm{min}$, detection at $280 \mathrm{~nm}$. Eluate fractions, containing putative 2a (RT $5 \mathrm{~min}$ ), 3a (RT $8 \mathrm{~min}$ ) and 5a (RT 17 min) were separately pooled. Next the eluent was changed to $80 \% \mathrm{MeCN}$ in water $+0.1 \%$ TFA and a peak containing 6a was collected. Freeze drying provided pure 2a (15 mg, 4 \%), 5a (156 mg, 33 \%) and partially purified 3a and $\mathbf{6 a}$ as powders. The fraction containing $\mathbf{3 a}$ was dissolved in $16 \% \mathrm{MeCN}$ in water and applied in several portions onto an HPLC column $(4 \times 250 \mathrm{~mm}$, Merck Hibar Lichrosorb $\mathrm{RP} 18,10 \mu \mathrm{m}$ ), eluent $-16 \% \mathrm{MeCN}$ in water $+0.1 \% \mathrm{TFA}$, flow $2 \mathrm{~mL} / \mathrm{min}$, detection at $220 \mathrm{~nm}$. Eluate fractions containing pure 3a were pooled and lyophilized to give a yellow powder. (yield $15 \mathrm{mg}, 4 \%$ ). The fraction containing 6a was purified again on the Vydac column (eluent - $22 \% \mathrm{MeCN}$ in water + $0.1 \%$ TFA), followed by purification on the Merck column (eluent $-22 \% \mathrm{MeCN}$ in water $+0.1 \%$ TFA) to give, after freeze drying, a white powder $(2.1 \mathrm{mg}, 0.3 \%)$.

\section{Characterization data}

2,3-Dihydro-2,3'-biindole-5,5'-dicarboxylic acid (2a): HRMS: $\mathrm{M}+\mathrm{H}^{+} \quad\left(\mathrm{C}_{18} \mathrm{H}_{15} \mathrm{~N}_{2} \mathrm{O}_{4}\right)$ 323.1019, calculated 323.1032; M-H $\left(\mathrm{C}_{18} \mathrm{H}_{13} \mathrm{~N}_{2} \mathrm{O}_{4}\right)$ 321.0861, calculated 321.0876; Elemental analysis: found, \%: N 8.2, C 64.9, H 4.8; calculated for $3 \mathrm{C}_{18} \mathrm{H}_{14} \mathrm{~N}_{2} \mathrm{O}_{4} \cdot 2 \mathrm{H}_{2} \mathrm{O}, \%$ : N 8.38, C 64.67, H 4.62.

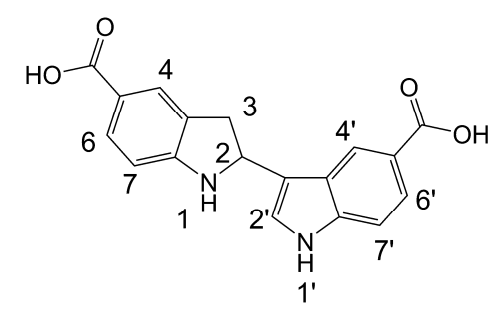


${ }^{1} \mathrm{H}-\mathrm{NMR}\left(400 \mathrm{MHz}, \mathrm{DMSO}_{6}, 2^{\circ} 5^{\circ} \mathrm{C}\right): \delta 3.03(\mathrm{dd}, J=16.0,8.8 \mathrm{~Hz}, 1 \mathrm{H}, \mathrm{H}-3), 3.43(\mathrm{dd}, J=16.0,9.2$ Hz, 1H, H-3), 5.28 (t, $J=9.2 \mathrm{~Hz}, 1 \mathrm{H}, \mathrm{H}-2), 6.50$ (d, $J=8.0 \mathrm{~Hz}, 1 \mathrm{H}, \mathrm{H}-7), 7.36$ (d, $J=2.0 \mathrm{~Hz}, 1 \mathrm{H}, \mathrm{H}-$ 4), $7.53(\mathrm{~d}, J=8.4 \mathrm{~Hz}, 1 \mathrm{H}), 7.58(\mathrm{~m}, 1 \mathrm{H}), 7.61$ (dd, $J=8.0,1.6 \mathrm{~Hz}, 1 \mathrm{H}, \mathrm{H}-6), 7.81$ (dd, $J=8.4,1.6$ $\mathrm{Hz}, 1 \mathrm{H}), 7.96$ (m, 1H), 11.26 (d, $J=1.6 \mathrm{~Hz}, 1 \mathrm{H}, \mathrm{H}-1), 11.79$ (d, $J=2.0 \mathrm{~Hz}, 1 \mathrm{H}, \mathrm{H}-1$ '), 12.3 (m, 2H, $2 \mathrm{COOH})$

3,3'-[2-(2-Amino-5-carboxyphenyl)ethane-1,1-diyl]bis(1H-indole-5-carboxylic acid (3a): HRMS: $\mathrm{M}+\mathrm{H}^{+}\left(\mathrm{C}_{27} \mathrm{H}_{22} \mathrm{~N}_{3} \mathrm{O}_{6}\right)$ 484.1504, calculated 484.1508; $\mathrm{M}-\mathrm{H}^{-}\left(\mathrm{C}_{27} \mathrm{H}_{20} \mathrm{~N}_{3} \mathrm{O}_{6}\right)$ 482.1331, calculated 482.1352; Elemental analysis: found, \%: N 7.7, C 58.5, H 5.4; calculated for $\mathrm{C}_{27} \mathrm{H}_{21} \mathrm{~N}_{3} \mathrm{O}_{6} \cdot 4 \mathrm{H}_{2} \mathrm{O}, \%$ : $\mathrm{N}$ 7.56, C 58.37, H 5.26.

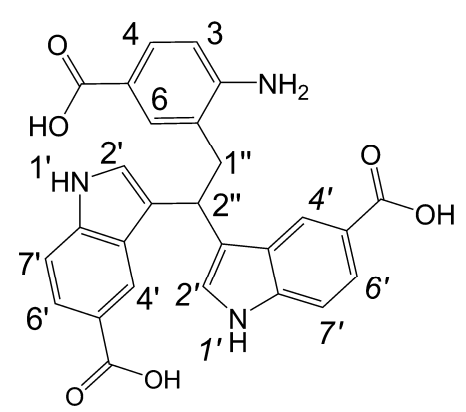

${ }^{1} \mathrm{H}-\mathrm{NMR}\left(270 \mathrm{MHz}, \mathrm{DMSO}-\mathrm{d}_{6}, 25^{\circ} \mathrm{C}\right): \delta 3.22\left(\mathrm{~m}, 2 \mathrm{H}, 2 \mathrm{H}-1{ }^{\prime \prime}\right), 4.93(\mathrm{~m}, 1 \mathrm{H}, \mathrm{H}-2$ '’), $6.58(\mathrm{~d}, J=8.6$ Hz, 1H, H-3), 7.31 (d, J=8.6 Hz, 2H, H-7', H-7'), 7.33, 7.34 (2m, 2H, H-2', H-2 '), 7.35 (m, 1H, H-4), 7.40 (m, 1H, H-6), 7.59 (dd, $J=8.6 \mathrm{~Hz}, 1.6 \mathrm{~Hz}, 2 \mathrm{H}, \mathrm{H}-6$ ', H-6'), 8.08 (d, J=1.7 Hz, 2H, H-4', H-4'), 11.14 (d, $J=1.9 \mathrm{~Hz}, 2 \mathrm{H}, \mathrm{H}-1$ ', H-1').

3-(2-(2-Amino-5-carboxyphenyl)-1-(2-mercaptoethylthio)ethyl)-1H-indole)-5-carboxylic acid (5a): HRMS: $\mathrm{M}+\mathrm{H}^{+}\left(\mathrm{C}_{20} \mathrm{H}_{21} \mathrm{~N}_{2} \mathrm{O}_{4} \mathrm{~S}_{2}\right)$ 417.0957, calculated 417.0943; $\mathrm{M}-\mathrm{H}^{-}\left(\mathrm{C}_{20} \mathrm{H}_{19} \mathrm{~N}_{2} \mathrm{O}_{4} \mathrm{~S}_{2}\right)$ 415.0794, calculated 415.0787; Elemental analysis: found, \%: N 6.5, C 57.8, H 5.0; calculated for $\mathrm{C}_{20} \mathrm{H}_{20} \mathrm{~N}_{2} \mathrm{O}_{4} \mathrm{~S}_{2}$, $\%$ : N 6.73, C 57.67, H 4.84.

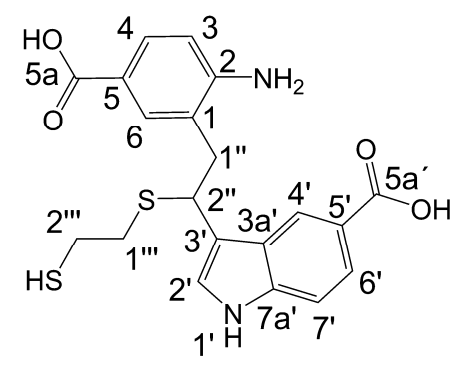

${ }^{1} \mathrm{H}-\mathrm{NMR}\left(400 \mathrm{MHz}, \mathrm{DMSO}-\mathrm{d}_{6}, 25^{\circ} \mathrm{C}\right): \delta 2.34(\mathrm{~m}, 1 \mathrm{H}, \mathrm{SH}), 2.5$ (m, 4H, 2H-1 $\left.{ }^{\prime \prime}, 2 \mathrm{H}-2{ }^{\prime \prime}{ }^{\prime}\right), 3.22(\mathrm{~m}, 2 \mathrm{H}$, 2H-1' '), 4.67(m, 1H, H-2'”), 6.61 (d, $J=8.3 \mathrm{~Hz}, 1 \mathrm{H}, \mathrm{H}-3), 7.39$ (d, $J=8.6 \mathrm{~Hz}, 1 \mathrm{H}, \mathrm{H}-7$ ') 7.45 (d, $J=$ $2.2 \mathrm{~Hz}, 1 \mathrm{H}, \mathrm{H}-2$ ') 7.46 (dd, $J=8.3 \mathrm{~Hz}, 2.0 \mathrm{~Hz}, 1 \mathrm{H}, \mathrm{H}-4), 7.53$ (d, $J=2.0 \mathrm{~Hz}, 1 \mathrm{H}, \mathrm{H}-6), 7.71$ (dd, $J=$ $8.6 \mathrm{~Hz}, 1.6 \mathrm{~Hz}, 1 \mathrm{H}, \mathrm{H}-6$ '), 8.43 (d, $J=1.6 \mathrm{~Hz}, 1 \mathrm{H}, \mathrm{H}-4$ '), 11.27 (d, J=2.2 Hz, 1H, H-1'); ${ }^{13} \mathrm{C}-\mathrm{NMR}$ (100 MHz, DMSO-d $6,25^{\circ} \mathrm{C}$ ): $\delta 24.8$ (C-2'”'), 35.0 (C-1'’'), 37.2 (C-1'’), 40.0 (C-2'’), 112.0 (C-7'), 116.6 (C-3'), 119.0 (C-5), 121.5 (C-5'), 122.8 (C-4'), 123.1 (C-1), 123.1 (C-6'), 125.9 (C-3a'), 126.0 (C-2’), 129.6 (C-4), 132.8 (C-6), 139.6 (C-7a'), 150.0 (C-2), 168.1 (C-5a), 169.1 (C-5a'). 
3,3'-\{1,1'-[Ethane-1,2-diylbis(sulfane-diyl)]bis[2-(2-amino-5-carboxyphenyl)ethane-1,1-diyl]\}bis(1Hindole-5-carboxylic acid) (6a): HRMS: $\mathrm{M}+\mathrm{H}^{+}\left(\mathrm{C}_{38} \mathrm{H}_{35} \mathrm{~N}_{4} \mathrm{O}_{8} \mathrm{~S}_{2}\right)$ 739.1880, calculated 739.1896; ${\mathrm{M}-\mathrm{H}^{-}}^{-}$ $\left(\mathrm{C}_{38} \mathrm{H}_{33} \mathrm{~N}_{4} \mathrm{O}_{8} \mathrm{~S}_{2}\right)$ 737.1733, calculated 737.1740; Elemental analysis: found, \%: $\mathrm{N} \mathrm{5.3,} \mathrm{C} \mathrm{47.9,} \mathrm{H} \mathrm{4.2;}$ calculated for $\mathrm{C}_{38} \mathrm{H}_{34} \mathrm{~N}_{4} \mathrm{O}_{8} \mathrm{~S}_{2} \cdot 2 \mathrm{CF}_{3} \mathrm{COOH} \cdot 5 \mathrm{H}_{2} \mathrm{O}, \%$ : N 5.30, C 47.73, H 4.39.

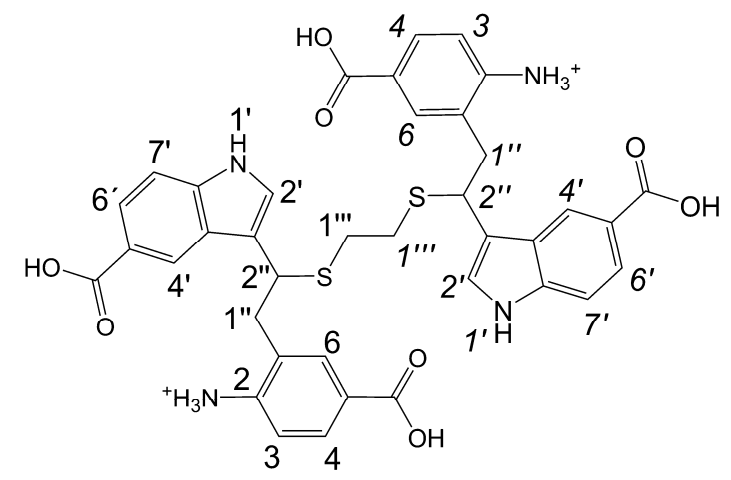

${ }^{1} \mathrm{H}-\mathrm{NMR}\left(270 \mathrm{MHz}\right.$, DMSO-d6, 25 $\left.{ }^{\circ} \mathrm{C}\right): \delta 2.3$ (m, 4H, 2H-1 ',', 2H-1 ','), 3.13 (m, 4H, 2H-1', $2 \mathrm{H}-1$ ' '), 4.55 (m, 2H, H-2', H-2 '), 6.55 (d, $J=8.6 \mathrm{~Hz}, 2 \mathrm{H}, \mathrm{H}-3, \mathrm{H}-3), 7.30,7.31$ (2d, J=2.3 Hz, 2H, H-2', H2'), 7.35 (d, $J=8.6 \mathrm{~Hz}, 2 \mathrm{H}, \mathrm{H}-7$ ', H-7'), 7.42-7.64 (m, 2H, H-4, H-4), 7.43-7.66 (m, 2H, H-6, H-6), 7.68 (dd, $J=8.6$ Hz, 1.6 Hz, 2H, H-6', H-6'), 8.38, 8.39 (2d, $J=1.6$ Hz, 2H, H-4', H-4'), 11.18 (d, $J=$ $2.3 \mathrm{~Hz}, 2 \mathrm{H}, \mathrm{H}-1$ ', H-1').

Procedure B: Compounds 2a, 3a, 5a and 6a were also obtained by a modification of Procedure A, whereby the crude product was dissolved in chloroform and applied onto a glass column filled with silica gel (pore size 60^, 70-230 mesh). The column was eluted with chloroform-methanol mixture, gradually changing its proportions from $20: 1$ to $1: 4$, and thereafter the elution was made with pure methanol. Eluate fractions containing pure 3a and 5a were separately pooled and evaporated. White crystalline products were obtained. Isolated yield of $\mathbf{3 a}$ was $7 \%$, and that of $\mathbf{5 a}$ was $25 \%$. (however, determination of the reaction products by HPLC provided the following yields: $2 \mathrm{a}: 22 \%, \mathbf{3 a}: 27 \%$, 5a: $28 \%$ and $6 \mathbf{a}: 1.3 \%)$.

3a: Elemental analysis. Found, \%: N 7.6, C 64.7, H 5.3. Calculated for $3 \mathrm{C}_{27} \mathrm{H}_{21} \mathrm{~N}_{3} \mathrm{O}_{4} \cdot 4 \mathrm{MeOH}, \%$ : $\mathrm{N}$ 7.99. C 64.67, H 5.04.

2-(2,2-bis(5-Chloro-1H-indol-3-yl)ethyl)-4-chloroaniline (3c). Compound 3c was obtained from 5chloroindole using Procedure A. Yield $63 \%$. HRMS: $\mathrm{M}+\mathrm{H}^{+}\left(\mathrm{C}_{24} \mathrm{H}_{19} \mathrm{Cl}_{3} \mathrm{~N}_{3}\right)$ 454.0637, calculated 454.0644; Elemental analysis: found, \%: $\mathrm{N}$ 7.17, $\mathrm{C}$ 54.81, $\mathrm{H}$ 3.45; calculated for $\mathrm{C}_{24} \mathrm{H}_{18} \mathrm{Cl}_{3} \mathrm{~N}_{3} \cdot \mathrm{CF}_{3} \mathrm{COOH}, \%$ : N 7.39, C 54.90, H 3.37.

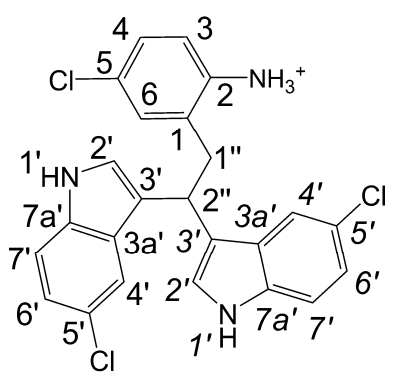


${ }^{1} \mathrm{H}-\mathrm{NMR}\left(400 \mathrm{MHz}, \mathrm{DMSO}_{\mathrm{d}}, 25^{\circ} \mathrm{C}\right): \delta 3.36(\mathrm{~d}, J=7.9 \mathrm{~Hz}, 2 \mathrm{H}, 2 \mathrm{H}-1$ ' ') 4.89 (t, $J=7.9 \mathrm{~Hz}, 1 \mathrm{H}, \mathrm{H}-$ 2'”), 6.78 (m, 1H, H-3), 6.93 (m, 2H, H-4, H-6), 6.99 (dd, J=8.6 Hz, 2.2 Hz, 2H, H-6', H-6'), 7.29 (d, $J=8.6$ Hz, 2H, H-7', H-7'), 7.42 (d, 2H, H-2', H-2 '), 7.49 (d, J=2.2 Hz, 2H, H-4', H-4 '), 10.99 (d, $J$ $\left.=2.4 \mathrm{~Hz}, 2 \mathrm{H}, \mathrm{H}-1^{\prime}, \mathrm{H}-1^{\prime}\right) ;{ }^{13} \mathrm{C}-\mathrm{NMR}\left(67.5 \mathrm{MHz}, \mathrm{DMSO}-\mathrm{d}_{6}, 25^{\circ} \mathrm{C}\right): \delta 32.0(\mathrm{C}-2$ '”), 35.7 (C-1'’), 113.4 (C-7', C-7'), 118.2 (C-3', C-3'), 118.8 (C-4', C-4'), 120.1 (C-3), 121.2 (C-6', C-6'), 123.3 (C-5', C5'), 124.9 (C-1, C-2', C-2 '), 126.8 (C-6), 128.2 (C-3a', C-3a'), 129.8 (C-4), 130.8 (C-5), 135.4 (C-7a', $\left.\mathrm{C}-7 a^{\prime}\right), 140.0(\mathrm{C}-2)$.

3-[2-(2-Amino-5-carboxyphenyl)-1-(phenethylthio)ethyl]-1H-indole-5-carboxylic acid (7a). This compound was prepared according to Procedure A using indole-5-carboxylic acid and 2phenylethanethiol as starting materials. Yield 19\%; HRMS: $\mathrm{M}+\mathrm{H}^{+}\left(\mathrm{C}_{26} \mathrm{H}_{25} \mathrm{~N}_{2} \mathrm{O}_{4} \mathrm{~S}\right) 461.1532$, calculated 461.1535; Elemental analysis: found, \%: $\mathrm{N}$ 5.82, $\mathrm{C}$ 65.44, $\mathrm{H} \mathrm{5.31;} \mathrm{calculated} \mathrm{for}$ $\mathrm{C}_{26} \mathrm{H}_{24} \mathrm{~N}_{2} \mathrm{O}_{4} \mathrm{~S} \cdot \mathrm{H}_{2} \mathrm{O}, \%$ : N 5.85, C 65.25, H 5.48.

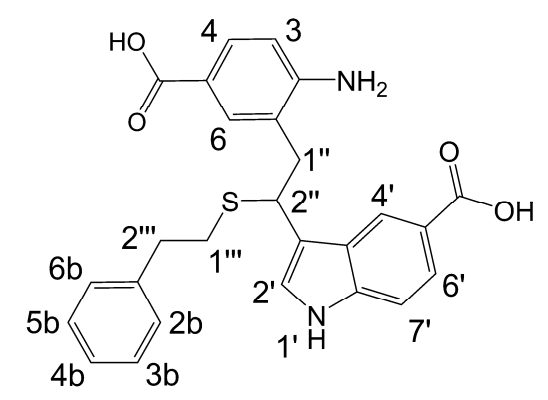

${ }^{1} \mathrm{H}-\mathrm{NMR}\left(270 \mathrm{MHz}, \mathrm{DMSO}-\mathrm{d}_{6}, 25^{\circ} \mathrm{C}\right): \delta 2.39$ and $2.58\left(2 \mathrm{~m}, 4 \mathrm{H}, 2 \mathrm{H}-1\right.$ ', $\left., 2 \mathrm{H}-2{ }^{\prime \prime}, '\right), 3.13-3.32(\mathrm{~m}, 2 \mathrm{H}$, 2H-1'), 4.66 (t, $\left.J=7.6 \mathrm{~Hz}, 1 \mathrm{H}, \mathrm{H}-2{ }^{\prime \prime}\right), 6.59$ (d, $\left.J=8.5 \mathrm{~Hz}, 1 \mathrm{H}, \mathrm{H}-3\right), 7.02$ (XX' part of AA'XX'system, 2H, H-2b, H-6b), 7.08-7.22 (m, 1H, H-4b), 7.08-7.22 (AA' part of AA'XX'system, 2H, H-3b, H-5b), 7.37 (d, J=8.6 Hz, 1H, H-7'), 7.45 (m, 2H, H-4, H-6), 7.53 (d, J=2.3 Hz, 1H, H2'), 7.69 (dd, $J=8.6 \mathrm{~Hz}, 1.6 \mathrm{~Hz}, 1 \mathrm{H}, \mathrm{H}-6$ '), 8.43 (d, $J=1.6 \mathrm{~Hz}, 1 \mathrm{H}, \mathrm{H}-4$ ') 11.28 (br s, 1H, H-1').

3-[2-(2-Amino-4-carboxyphenyl)-1-(phenethylthio)ethyl]-1H-indole-6-carboxylic acid (7b). Prepared according to Procedure A from indole-6-carboxylic acid and 2-phenylethanethiol. Yield 32\%; HRMS: $\mathrm{M}+\mathrm{H}^{+}\left(\mathrm{C}_{26} \mathrm{H}_{25} \mathrm{~N}_{2} \mathrm{O}_{4} \mathrm{~S}\right)$ 461.1548; calculated 461.1535. Elemental analysis: found, \%: N 5.26, C 61.88, $\mathrm{H}$ 4.74; calculated for $2 \mathrm{C}_{26} \mathrm{H}_{24} \mathrm{~N}_{2} \mathrm{O}_{4} \mathrm{~S} \cdot \mathrm{CF}_{3} \mathrm{COOH} \cdot \mathrm{H}_{2} \mathrm{O}, \%$ : N 5.32, C 61.59, H 4.88.

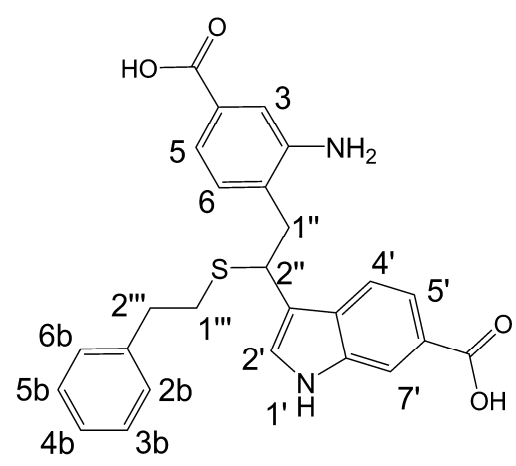

${ }^{1} \mathrm{H}-\mathrm{NMR}\left(400 \mathrm{MHz}, \mathrm{DMSO}_{\mathrm{d}}, 25^{\circ} \mathrm{C}\right): \delta 2.42$ and 2.52-2.63 (2m, 4H, 2H-1',', 2H-2','), 3.20-3.32 (m, 2H, 2H-1' '), 4.69 (m, 1H, H-2'’), 6.97 (d, J=8.0 Hz, 1H, H-H-6), 7.02 (XX' part of AA'XX'system, 2H, H-2b, H-6b), 7.02 (m, 1H, H-5), 7.09-7.14 (m, 1H, H-4b), 7.16-7.20 (AA' part of AA'XX'system, 
2H, H-3b, H-5b), 7.29 (d, $J=1.6 \mathrm{~Hz}, 1 \mathrm{H}, \mathrm{H}-3$ ), 7.53 (d, $J=2.4 \mathrm{~Hz}, 1 \mathrm{H}, \mathrm{H}-2$ ') 7.58 (dd, $J=8.4,1.6$ Hz, 1H, H-5'), 7.77 (d, $J=8.4$ Hz, 1H, H-4'), 7.96 (dd, $J=1.6,0.8$ Hz, 1H, H-7'), 11.26 (d, $J=2.8$ $\left.\mathrm{Hz}, 1 \mathrm{H}, \mathrm{H}-1^{\prime}\right)$.

3-\{2-[2-Amino-5-(diethylcarbamoyl)phenyl]-1-(phenethylthio)ethyl\}-N,N-diethyl-1H-indole-5-carboxamide (7c). Compound 7c was prepared according to Procedure A using indole-5-carboxylic acid diethylamide [24] and 2-phenylethanethiol as starting materials. Yield 30\%; $\mathrm{HRMS}: \mathrm{M}+\mathrm{H}^{+}$ $\left(\mathrm{C}_{34} \mathrm{H}_{43} \mathrm{~N}_{4} \mathrm{O}_{3} \mathrm{~S}\right)$ 571.3101; calculated 571.3106; Elemental analysis: found, \%: N 9.47, C 69.97, H 7.37; calculated for $6 \mathrm{C}_{34} \mathrm{H}_{42} \mathrm{~N}_{4} \mathrm{O}_{2} \mathrm{~S} \cdot \mathrm{CF}_{3} \mathrm{COOH}, \%$ : N 9.50, C 69.92, H 7.21.

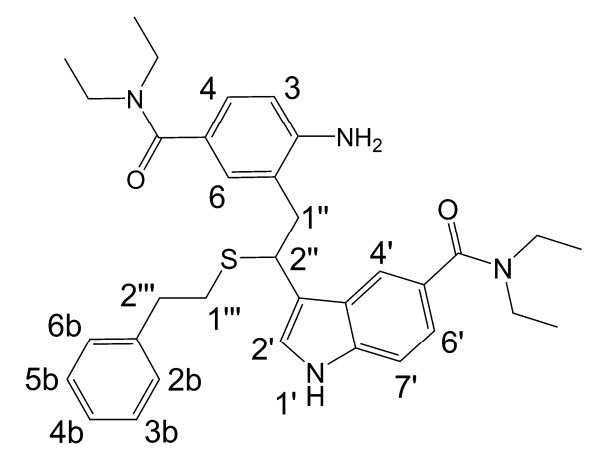

${ }^{1} \mathrm{H}-\mathrm{NMR}\left(400 \mathrm{MHz}, \mathrm{DMSO}-\mathrm{d}_{6}, 25^{\circ} \mathrm{C}\right): \delta 0.87\left(\mathrm{~m}, 6 \mathrm{H}, 2 \mathrm{CH}_{3}\right), 1.09\left(\mathrm{~m}, 6 \mathrm{H}, 2 \mathrm{CH}_{3}\right), 2.52-2.63(\mathrm{~m}, 4 \mathrm{H}$,

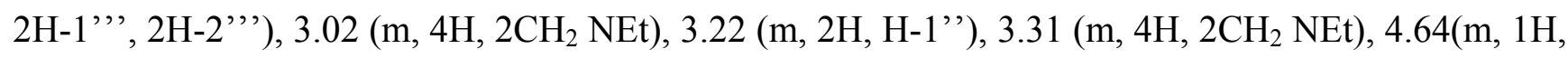
H-2' '), 6.69 (d, $J=8.0 \mathrm{~Hz}, 1 \mathrm{H}, \mathrm{H}-3), 6.77$ (d, $J=2.0 \mathrm{~Hz}, 1 \mathrm{H}, \mathrm{H}-6), 6.91$ (dd, $J=8.0,2.0 \mathrm{~Hz}, 1 \mathrm{H}, \mathrm{H}-$ 4), 7.00 (XX' part of AA'XX' system, 2H, H-2b, H-6b), 7.04 (dd, J=8.4, 1.6 Hz, 1H, H-6'), 7.09-7.13 (m, 1H, 1H-4b), 7.15-7.19 (AA' part of AA'XX'system, 2H, H-3b, H-5b), 7.33 (d, J=2.4 Hz, 1H, H2'), 7.34 (d, J=8.4 Hz, 1H, H-7'), 7.71 (m, 1H, 1H-4'), 11.08 (d, J=2.4 Hz, 1H, H-1').

3-[2-(2-Amino-5-cyanophenyl)-1-(phenethylthio)ethyl]-1H-indole-5-carbonitrile $\quad(7 \mathbf{d}) . \quad 7 \mathbf{d} \quad$ was prepared according to Procedure A from 5-cyanoindole and 2-phenylethanethiol. Yield 15\%; HRMS: $\mathrm{M}+\mathrm{H}^{+}\left(\mathrm{C}_{26} \mathrm{H}_{23} \mathrm{~N}_{4} \mathrm{~S}\right)$ 423.1674; calculated 423.1643.

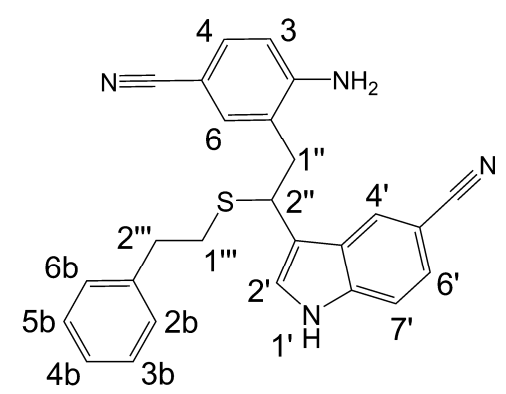

${ }^{1} \mathrm{H}-\mathrm{NMR}\left(400 \mathrm{MHz}, \mathrm{DMSO}-\mathrm{d}_{6}, 25^{\circ} \mathrm{C}\right): \delta 2.43$ and 2.52-2.64 (2m, 4H, 2H-1',', 2H-2','), 3.16 (dd, $J=$ 8.0, $2.0 \mathrm{~Hz}, 2 \mathrm{H}, 2 \mathrm{H}-1$ ' '), 4.70 (t, $J=8.0 \mathrm{~Hz}, 1 \mathrm{H}, \mathrm{H}-2$ ' '), 6.59 (d, $J=8.8 \mathrm{~Hz}, 1 \mathrm{H}, \mathrm{H}-3$ ), 7.02 (XX' part of AA'XX'system, 2H, H-2b, H-6b), 7.02 (m, 1H), 7.12-7.23 (AA' part of AA'XX'system, 2H, H-3b, H-5b), 7.12-7.23 (m, 1H, H-4b), 7.32 (d, $J=2.0 \mathrm{~Hz}, 1 \mathrm{H}), 7.36-7.44(\mathrm{~m}, 1 \mathrm{H}), 7.47$ (m, 1H), $7.54(\mathrm{~d}, J=$ $2.4 \mathrm{~Hz}, 1 \mathrm{H}), 11.48$ (br s, 1H, H-1'). 
4-Fluoro-2-[2-(5-fluoro-1H-indol-3-yl)-2-(phenethylthio)ethyl]aniline (7e). 7e was prepared according to Procedure A using 5-fluoroindole and 2-phenylethanethiol as starting materials. Yield 2\%; HRMS: $\mathrm{M}+\mathrm{H}^{+}\left(\mathrm{C}_{24} \mathrm{H}_{23} \mathrm{~F}_{2} \mathrm{~N}_{2} \mathrm{~S}\right)$ 409.1533; calculated 409.1550.

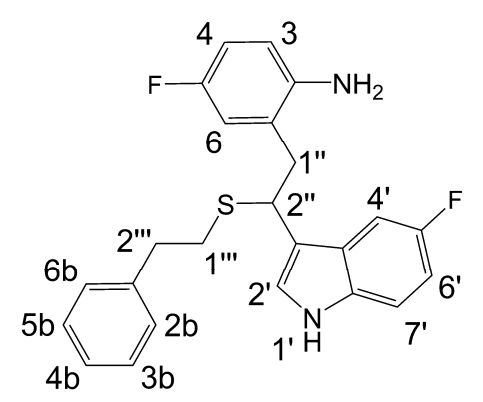

4-Chloro-2-[2-(5-chloro-1H-indol-3-yl)-2-(phenethylthio)ethyl]aniline (7f). 7f was prepared according to Procedure A from 5-chloroindole and 2-phenylethanethiol. Yield 5\%; $\mathrm{HRMS}: \mathrm{M}+\mathrm{H}^{+}$ $\left(\mathrm{C}_{24} \mathrm{H}_{23} \mathrm{Cl}_{2} \mathrm{~N}_{2} \mathrm{~S}\right)$ 441.0958; calculated 441.0959; Elemental analysis: found, \%: $\mathrm{N}$ 6.09, $\mathrm{C} \mathrm{65.43,} \mathrm{H}$ 4.96; calculated for $\mathrm{C}_{24} \mathrm{H}_{22} \mathrm{Cl}_{2} \mathrm{~N}_{2} \mathrm{~S}, \%$ : N 6.35, C 65.30, H 5.02.

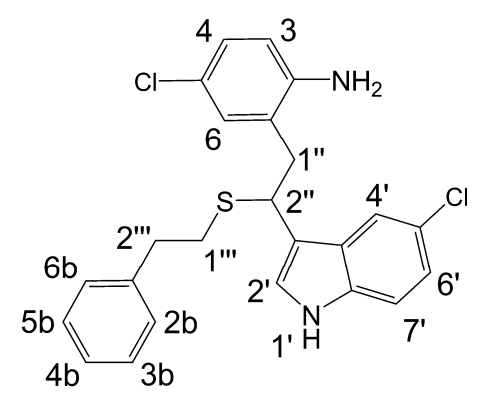

${ }^{1} \mathrm{H}-\mathrm{NMR}\left(400 \mathrm{MHz}, \mathrm{DMSO}-\mathrm{d}_{6}, 25^{\circ} \mathrm{C}\right): \delta 2.47$ and $2.53\left(2 \mathrm{~m}, 2 \mathrm{H}, 2 \mathrm{H}-1{ }^{\prime \prime \prime}\right), 2.62\left(\mathrm{~m}, 2 \mathrm{H}, 2 \mathrm{H}-2{ }^{\prime \prime \prime}\right), 3.14$ (m, 2H, 2H-1' '), 4.62 (m, 1H, 1H-2') ), 6.56 (d, $J=8.8 \mathrm{~Hz}, 1 \mathrm{H}, \mathrm{H}-3), 6.83$ (dd, $J=8.8,2.8 \mathrm{~Hz}, 1 \mathrm{H}, \mathrm{H}-$ 4), 6.91 (d, $J=2.8 \mathrm{~Hz}, 1 \mathrm{H}, \mathrm{H}-6), 7.02-7.06$ (XX' part of AA'XX'system, 2H, H-2b, H-6b), 7.02-7.06 (m, 1H, H-6'), 7.12 (m, 1H, H-4b), 7.17-7.22 (AA' part of AA'XX'system, 2H, H-3b, H-5b), 7.32 (d, $J$ $\left.=8.4 \mathrm{~Hz}, 1 \mathrm{H}, \mathrm{H}-7^{\prime}\right), 7.41$ (d, $\left.J=2.4 \mathrm{~Hz}, 1 \mathrm{H}, \mathrm{H}-2^{\prime}\right), 7.73$ (d, J=2.0 Hz, 1H, H-4'), 11.09 (br s, 1H, H1 ').

3-[2-(2-Aminophenyl)-1-(phenethylthio)ethyl]-1H-indole-5-carboxylic acid (9). Compound 9 was prepared according to Procedure A using indole, indole-5-carboxylic acid (equimolar quantities) and 2-phenylethanethiol as starting materials. Yield 19\%; HRMS: $\mathrm{M}+\mathrm{H}^{+}$417.1618. $\mathrm{C}_{25} \mathrm{H}_{25} \mathrm{~N}_{2} \mathrm{O}_{2} \mathrm{~S}$; calculated 417.1636. Elemental analysis: found, \%: $\mathrm{N} 5.94, \mathrm{C} 64.41, \mathrm{H}$ 5.10; calculated for $2 \mathrm{C}_{25} \mathrm{H}_{24} \mathrm{~N}_{2} \mathrm{O}_{2} \mathrm{~S} \cdot \mathrm{CF}_{3} \mathrm{COOH} \cdot \mathrm{H}_{2} \mathrm{O}, \%$ : N 5.81, C 64.71, H 5.33.

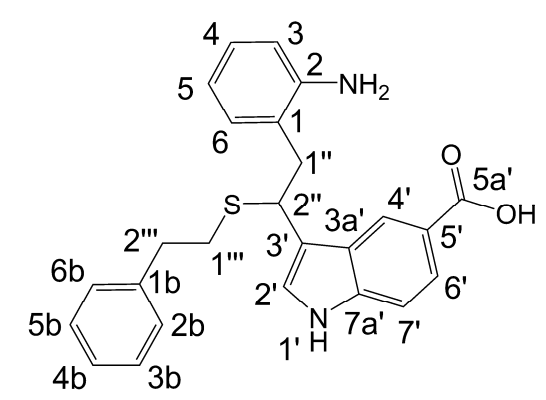


${ }^{1} \mathrm{H}-\mathrm{NMR}\left(500 \mathrm{MHz}, \mathrm{DMSO}-\mathrm{d}_{6}, 2^{\circ} \mathrm{C}\right): \delta 2.65$ (m, 2H, 2H-2",'), 3.31, 3.35 (m, 2H, 2H-1"',), 3.31, 3.35 (m, 2H, 2H-1'’), 4.74 (m, 1H, H-2'’), 6.79 (m, 1H, H-3), 6.96(m, 1H, H-5), 7.03 (m, 1H, H-6), 7.05 (m, 1H, H-4), 7.05 (XX' part of AA'XX'system, 2H, H-2b, H-6b), 7.14 (m, 1H, H-4b), 7.20 (AA' part of AA'XX'system, 2H, H-3b, H-5b), 7.40 (d, $J=8.6 \mathrm{~Hz}, 1 \mathrm{H}, \mathrm{H}-7^{\prime}$ ), 7.46 (d, $J=2.3 \mathrm{~Hz}, 1 \mathrm{H}, \mathrm{H}-$ 2'), 7.71 (dd, $J=8.6 \mathrm{~Hz}, 1.5 \mathrm{~Hz}, 1 \mathrm{H}, \mathrm{H}-6$ '), 8.46 (s, 1H, H-4'), 11.28 (d, $\left.J=1.8 \mathrm{~Hz}, 1 \mathrm{H}, \mathrm{H}-1^{\prime}\right) .{ }^{13} \mathrm{C}$ NMR (125 MHz, DMSO-d $\left.6,25^{\circ} \mathrm{C}\right): \delta 31.1$ (C-1','), 34.9 (C-2','), $35.6\left(\mathrm{C}-1{ }^{\prime \prime}\right), 38.7$ (C-2'”), 110.6 (C7'), 115.2 (C-3'), 118.4 (C-5), 120.2 (C-5'), 121.1 (C-3), 121.4 (C-6'), 121.8 (C-4'), 124.6 (C-2'), 124.7 (C-3a'), 125.2 (C-4b), 126.4 (C-4), 127.4 (C-3b, C-5b), 127.6 (C-2b, C-6b), 129.8 (C-6), 129.9 (C-1), 138.2 (C-7a'), 139.8 (C-1b), 150.0 (C-2), 167.7 (C-5a').

3-[2-(2-Aminophenyl)-1-(phenethylthio)ethyl]-2,3-dihydro-1H,1'H-2,3'-biindole-5'-carboxylic acid (10). This compound was prepared according to Procedure A using indole, indole-5-carboxylic acid (equimolar quantities) and 2-phenylethanethiol. Yield 1\%; HRMS: $\mathrm{M}+\mathrm{H}^{+}\left(\mathrm{C}_{33} \mathrm{H}_{32} \mathrm{~N}_{3} \mathrm{O}_{2} \mathrm{~S}\right)$ 534.2212; calculated 534.2215.

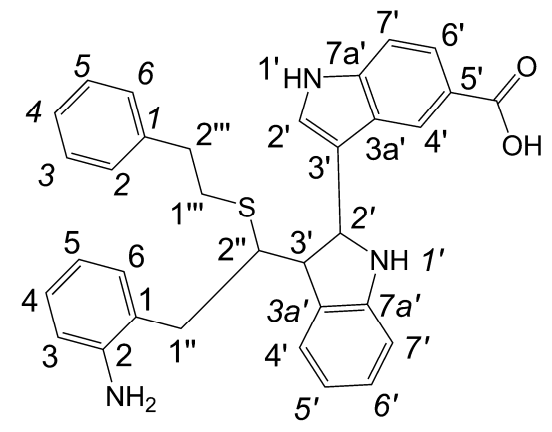

${ }^{1} \mathrm{H}-\mathrm{NMR}\left(500 \mathrm{MHz}, \mathrm{DMSO}_{6}, \mathrm{~d}_{6}^{\circ} \mathrm{C}\right): \delta 2.23$ (m, 2H, 2H-1 $\left.{ }^{\prime \prime \prime}\right), 2.30,2.37$ (m, 2H, 2H-2','), 2.65 (m, $1 \mathrm{H}, \mathrm{H}_{\mathrm{a}}-1$ ' ) 2.94 (m, 1H, $\mathrm{H}_{\mathrm{b}}-1$ '’), 3.54 (m, 1H, H-2'”), 3.98 (m, 1H, H-3 '), 5.24 (d, J= 7.6 Hz, 1H, H2'), 6.64 (d, $J=7.5 \mathrm{~Hz}, 1 \mathrm{H}, \mathrm{H}-7$ ') 6.71 (m, 1H, H-5'), 6.72 (m, 1H, H-3), 6.78 (XX' part of AA'XX'system, 2H, H-2, H-6), 6.91(m, 1H, H-5), 6.93 (m, 1H, H-4), 7.05 (m, 1H, H-6), 7.06 (m, 1H, H-6'), 7.11 (AA' part of AA'XX'system, 2H, H-3, H-5), 7.14 (m, 1H, H-4), 7.31 (d, J=7.3 Hz, 1H, H-4'), 7.35 (m, 1H, H-2'), 7.45 (d, $J=8.6 \mathrm{~Hz}, 1 \mathrm{H}, \mathrm{H}-7^{\prime}$ ), 7.74 (dd, $J=8.6 \mathrm{~Hz}, 1.5 \mathrm{~Hz}, 1 \mathrm{H}, \mathrm{H}-6$ '), 8.26 (s, 1H, H-4'), 11.35 (m, 1H, H-1'), 12.36 (br, 1H, COOH); ${ }^{13} \mathrm{C}-\mathrm{NMR}$ (125 MHz, DMSO-d $6,25^{\circ} \mathrm{C}$ ):

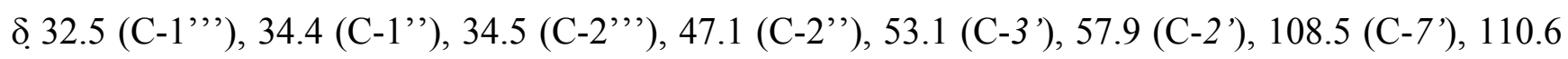
(C-7'), 117.2 (C-5'), 117.5 (C-5), 117.8 (C-3'), 120.3 (C-5'), 121.6 (C-4'), 121.8 (C-6'), 123.7 (C-4'), 124.4 (C-2'), 124.5 (C-3a'), 125.2 (C-4), 126.9 (C-6), 126.9 (C-6'), 127.3 (C-3, 5), 127.5 (C-2, 6), 130.5 (C-4), 138.6 (C-7a'), $139.6(\mathrm{C}-1), 167.5$ (C-COOH).

3-[2-(2-Aminophenyl)-1-(phenethylthio)ethyl]-1H,1'H-2,3'-biindole-5'-carboxylic acid (11). Compound 11 was synthesized from $10(5 \mathrm{mg})$ when the latter was dissolved in DMSO-d $\mathrm{d}_{6}(0.7 \mathrm{~mL})$ and the solution was allowed to stand in a NMR tube at room temperature for one week. 


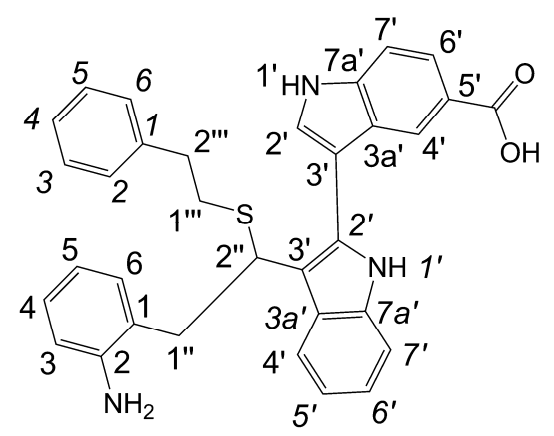

${ }^{1} \mathrm{H}-\mathrm{NMR}\left(500 \mathrm{MHz}, \mathrm{DMSO}-\mathrm{d}_{6}, 25^{\circ} \mathrm{C}\right): \delta 2.21,2.30\left(\mathrm{~m}, 4 \mathrm{H}, 2 \mathrm{H}-1{ }^{\prime \prime},{ }^{2}, 2 \mathrm{H}-2{ }^{\prime \prime}{ }^{\prime}\right), 3.39\left(\mathrm{~m}, 1 \mathrm{H}, \mathrm{H}_{\mathrm{a}}-1{ }^{\prime \prime}\right)$ ), 3.45 (m, 1H, $\mathrm{H}_{\mathrm{b}}-1$ ') $), 4.64$ (dd, $\left.J=8.9 \mathrm{~Hz}, 5.9 \mathrm{~Hz}, 1 \mathrm{H}, \mathrm{H}-2{ }^{\prime \prime}\right), 6.55$ (XX' part of AA'XX'system, 2H, H-2, H-6), 6.70 (d, $J=7.4 \mathrm{~Hz}, 1 \mathrm{H}, \mathrm{H}-6), 6.80$ (m, 1H, H-2'), 6.94 (m, 1H, H-4), 6.96 (AA' part of AA'XX'system, 2H, H-3, H-5), 7.03 (m, 1H, H-5), 7.03 (m, 1H, H-4), 7.07 (m, 1H, H-5'), 7.13 (m, 1H, H-6'), 7.42 (d, J=8.0 Hz, 1H, H-7'), 7.48 (d, J=8.6 Hz, 1H, H-7'), 7.79 (dd, J=8.6 Hz, $1.5 \mathrm{~Hz}$, $1 \mathrm{H}, \mathrm{H}-6$ ') 8.02 (d, J=7.9 Hz, 1H, H-4'), 8.36 (s, 1H, H-4'), 11.20 (m, 1H, H-1 '), 11.74 (m, 1H, H1'), 12.50 (br, 1H, COOH); ${ }^{13} \mathrm{C}-\mathrm{NMR}\left(125 \mathrm{MHz}, \mathrm{DMSO}-\mathrm{d}_{6}, 25^{\circ} \mathrm{C}\right): \delta 31.5$ (C-1 ', '), 35.1 (C-1 ', 2','), 39.9 (C-2'’), 107.9 (C-3'), 109.6 (C-3'), 110.7 (C-7', 7'), 117.7 (C-5'), 119.4 (C-4'), 120.2 (C-6'), 121.4 (C-5'), 121.9 (C-4'), 122.3(C-6'), 125.0 (C-4), 125.2 (C-3a'), 125.3 (C-2'), 125.5 (C-3a'), 126.8 (C-4, 5), 127.2 (C-3, 5), 127.3 (C-2, 6), 129.6 (C-2), 129.7 (C-6), 130.6 (C-1, 2'), 136.5 (C-7a'), 137.9 (C-7a'), 139.4 (C-1), 167.5 (C-COOH).

Procedure C. Preparation of 3,3'-\{1,1'-[ethane-1,2-diylbis(sulfanediyl)]bis[2-(2-acetamido-5carboxyphenyl)ethane-1,1-diyl]\}bis(1H-indole-5-carboxylic acid) (6b). Compound 6a (6.6 mg, 6.24 $\mu \mathrm{mol})$ was dissolved in DMF $(180 \mu \mathrm{L})$ and acetic anhydride $(30 \mu \mathrm{L}, 318 \mu \mathrm{mol})$ was added. The mixture was allowed to stand for $40 \mathrm{~h}$, diluted with water to $1 \mathrm{~mL}$ volume, centrifuged and the clear solution in several portions applied onto an HPLC semipreparative column (10 x $250 \mathrm{~mm}$, Vydac RP $\mathrm{C}_{18} 90 \AA$ Pharmaceutical 201HS1010), eluent $-22 \% \mathrm{MeCN}$ in water $+0.1 \% \mathrm{TFA}$, flow $5 \mathrm{~mL} / \mathrm{min}$, detection at $280 \mathrm{~nm}$. Eluate fractions containing pure putative $\mathbf{6 b}$ were pooled and freeze-dried. Yield ofoff-white powder was $3.4 \mathrm{mg}$ (57\%); HRMS: $\mathrm{M}+\mathrm{H}^{+}\left(\mathrm{C}_{42} \mathrm{H}_{39} \mathrm{~N}_{4} \mathrm{O}_{10} \mathrm{~S}_{2}\right)$ 823.2129; calculated

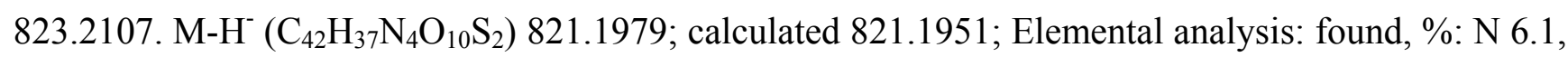
C 54.3, H 4.6; calculated for $3 \mathrm{C}_{42} \mathrm{H}_{38} \mathrm{~N}_{4} \mathrm{O}_{10} \mathrm{~S}_{2} \cdot 2 \mathrm{CF}_{3} \mathrm{COOH} \cdot 9 \mathrm{H}_{2} \mathrm{O}, \%$ : N 5.88. C 54.62, H 4.72.

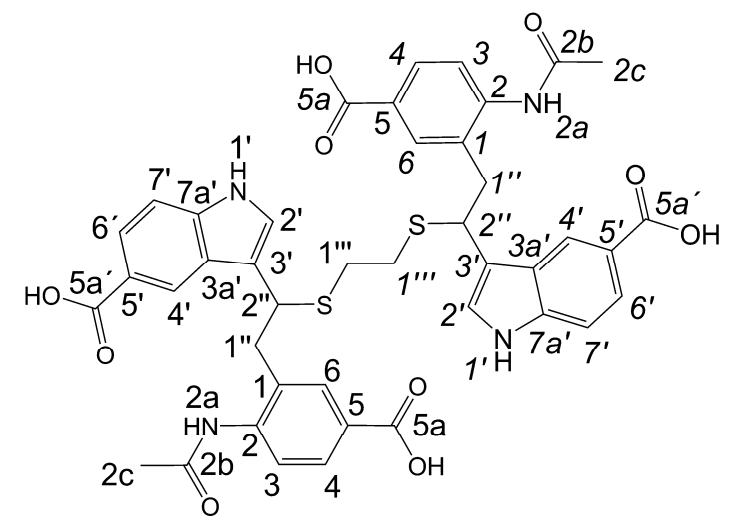

${ }^{1} \mathrm{H}-\mathrm{NMR}\left(500 \mathrm{MHz}, \mathrm{DMSO}_{\mathrm{d}}, 25^{\circ} \mathrm{C}\right): \delta 1.88,1.90$ (2s, 6H, H-2c, H-2c), $2.3-2.5$ (m, 4H, 2H-1",', 2H-1 ','), 3.25 - 3.40 (m, 4H, 2H-1', 2H-1' '), 4.42, 4.44 (2m, 2H, H-2' ', H-2 ' ') 7.19 (m, 2H, H-2', H2'), 7.33 (dd, $J=8.5 \mathrm{~Hz}, 2.7 \mathrm{~Hz}, 2 \mathrm{H}, \mathrm{H}-7$ ', H-7'), 7.45 (d, J=8.6 Hz, 2H, H-3, H-3), 7.64 (m, 2H, H- 
4, H-4), 7.66 (m, 2H, H-6, H-6), 7.68 (m, 2H, H-6', H-6'), 8.38, 8.39 (2m, 2H, H-4', H-4'), 9.35, 9.36 (2s, 2H, H-2a, H-2a), 11.14 (m, 2H, H-1', H-l'), 12.5 (br, 2H, H-5a, H-5a); ${ }^{13} \mathrm{C}$ NMR (125 MHz,

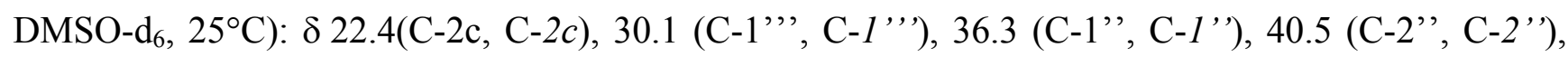
110.6 (C-7', C-7'), 114.5 (C-3', C-3 '), 120.4 (C-5', C-5'), 121.6 (C-4', C-4'), 126.1 (C-5, C-5), 121.8 (C-6', C-6'), 124.3 (C-3a', C-3a'), 124.6 (C-2', C-2'), 126.9 (C-4, C-4), 131.0 (C-6, C-6), 132.3 (C-1, C-1), 138.6 (C-7a', C-7a'), 140.0 (C-2, C-2), 166.2 (C-5a, C-5a), 167.8 (C-5a', C-5a'), 167.8 (C-2b, $\mathrm{C}-2 b$ ).

The following compounds were similarly prepared by Procedure C:

1-Acetyl-2,3-dihydro-1H,1'H-2,3'-biindole-5,5'-dicarboxylic acid (2b). From 2a. HRMS: $\mathrm{M}+\mathrm{H}^{+}$ $\left(\mathrm{C}_{20} \mathrm{H}_{17} \mathrm{~N}_{2} \mathrm{O}_{5}\right)$ 365.1138; calculated 365.1137; $\mathrm{M}-\mathrm{H}^{-}\left(\mathrm{C}_{20} \mathrm{H}_{15} \mathrm{~N}_{2} \mathrm{O}_{5}\right)$ 363.0961; calculated 363.0981; Elemental analysis: found, \%: N 6.3, C 56.1, H 4.2; calculated for $2 \mathrm{C}_{20} \mathrm{H}_{16} \mathrm{~N}_{2} \mathrm{O}_{5} \cdot \mathrm{CF}_{3} \mathrm{COOH} \cdot 3 \mathrm{H}_{2} \mathrm{O}, \%$ : N 6.25. C 56.25, H 4.38.

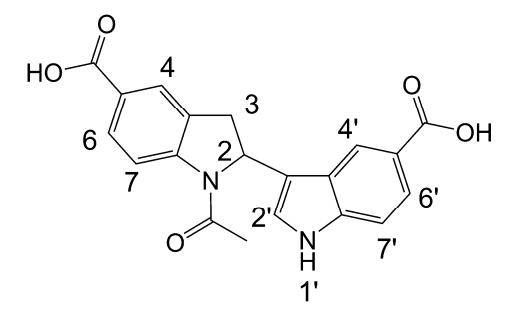

${ }^{1} \mathrm{H}-\mathrm{NMR}\left(400 \mathrm{MHz}, \mathrm{DMSO}-\mathrm{d}_{6}, 25^{\circ} \mathrm{C}\right): \delta 2.07\left(\mathrm{~s}, 3 \mathrm{H}, \mathrm{CH}_{3}\right), 3.01(\mathrm{~d}, J=16.0 \mathrm{~Hz}, 1 \mathrm{H}, \mathrm{H}-3), 3.79$ (dd, $J$ $=16.0,10.4 \mathrm{~Hz}, 1 \mathrm{H}, \mathrm{H}-3), 6.04$ (dd, $J=10.0,2.0 \mathrm{~Hz}, 1 \mathrm{H}, \mathrm{H}-2), 7.17$ (d, J=2.0 Hz, 1H, H-2'), 7.40 (d, $J=8.8 \mathrm{~Hz}, 1 \mathrm{H}, \mathrm{H}-7), 7.69$ (dd, $J=8.8,1.6 \mathrm{~Hz}, 1 \mathrm{H}, \mathrm{H}-6), 7.77$ (d, $J=1.6 \mathrm{~Hz}, 1 \mathrm{H}, \mathrm{H}-4), 7.88$ (dd, $J=$ 8.4, 2.0 Hz, 1H, H-6'), 7.98 (s, 1H, H-4'), 8.18 (m, 1H, H-7'), 11.34 (br s, 1H, H-1').

3,3'-[2-(2-Acetamido-5-carboxyphenyl)ethane-1,1-diyl]bis(1H-indole-5-carboxylic acid) (3b). From 3a. HRMS: $\mathrm{M}+\mathrm{H}^{+}\left(\mathrm{C}_{29} \mathrm{H}_{24} \mathrm{~N}_{3} \mathrm{O}_{7}\right)$ 526.162, calculated 526.1614. $\mathrm{M}-\mathrm{H}^{-}\left(\mathrm{C}_{29} \mathrm{H}_{22} \mathrm{~N}_{3} \mathrm{O}_{7}\right)$ 524.1467, calculated 524.1458; Elemental analysis: found, \%: $\mathrm{N}$ 6.2, $\mathrm{C}$ 53.4, $\mathrm{H}$ 4.5; calculated for $\mathrm{C}_{29} \mathrm{H}_{23} \mathrm{~N}_{3} \mathrm{O}_{4} \cdot \mathrm{CF}_{3} \mathrm{COOH} \cdot 3 \mathrm{H}_{2} \mathrm{O}, \%$ : N 6.06. C 53.68, $\mathrm{H} 4.36$.

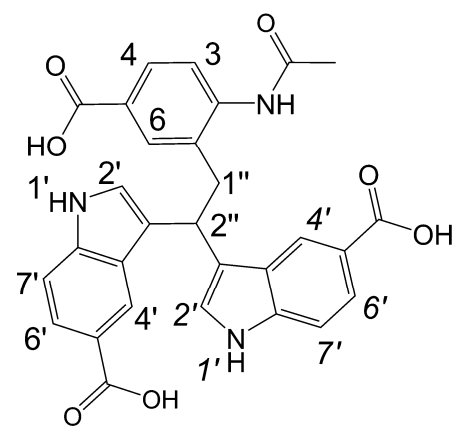

${ }^{1} \mathrm{H}-\mathrm{NMR}\left(400 \mathrm{MHz}, \mathrm{DMSO}-\mathrm{d}_{6}, 25^{\circ} \mathrm{C}\right): \delta 1.88\left(\mathrm{~s}, 3 \mathrm{H}, \mathrm{CH}_{3}\right), 3.54(\mathrm{~d}, J=7.6 \mathrm{~Hz}, 2 \mathrm{H}, 2 \mathrm{H}-1$ '’), 4.83 (dd, $J=7.6,7.6 \mathrm{~Hz}, 1 \mathrm{H}, 1 \mathrm{H}-2^{\prime}$ ') 7.27 (d, $J=2.4 \mathrm{~Hz}, 2 \mathrm{H}, \mathrm{H}-1$ ', H-1 '), 7.31 (d, J=8.8 Hz, 2H, H-7', H-7'), 7.48 (d, $J=8.4 \mathrm{~Hz}, 1 \mathrm{H}, \mathrm{H}-3), 7.59$ (dd, $J=8.8,1.6 \mathrm{~Hz}, 2 \mathrm{H}, \mathrm{H}-6$ ', H-6'), 7.61 (dd, $J=8.4,2.0 \mathrm{~Hz}, 1 \mathrm{H}$, H-4), 7.69 (d, $J=2.0 \mathrm{~Hz}, 1 \mathrm{H}, \mathrm{H}-6), 8.11$ (d, $J=1.6 \mathrm{~Hz}, 2 \mathrm{H}, \mathrm{H}-4$ ', H-4 '), 9.31 (s, 1H, H-2a), 11.13 (d, $J=2.4 \mathrm{~Hz}, 2 \mathrm{H}, \mathrm{H}-1^{\prime}, H-1$ '). 
3-[2-(2-Acetamido-5-carboxyphenyl)-1-(2-mercaptoethylthio)ethyl]-1H-indole-5-carboxylic acid (5b). From 5a. HRMS: $\mathrm{M}+\mathrm{H}^{+}\left(\mathrm{C}_{22} \mathrm{H}_{23} \mathrm{~N}_{2} \mathrm{O}_{5} \mathrm{~S}_{2}\right)$ 459.1037; calculated 459.1048; Elemental analysis: found, \%: N 5.70, C 56.39, H 4.81; calculated for $8 \mathrm{C}_{22} \mathrm{H}_{22} \mathrm{~N}_{2} \mathrm{O}_{5} \mathrm{~S}_{2} \cdot \mathrm{CF}_{3} \mathrm{COOH}, \%$ : N 5.92. C 56.52, H 4.72.

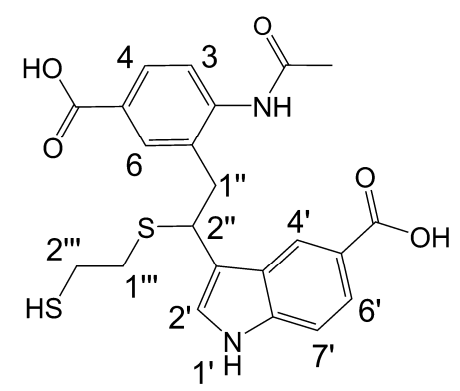

${ }^{1} \mathrm{H}-\mathrm{NMR}\left(400 \mathrm{MHz}, \mathrm{DMSO}_{-} \mathrm{d}_{6}, 25^{\circ} \mathrm{C}\right): \delta 1.95\left(\mathrm{~s}, 3 \mathrm{H}, \mathrm{CH}_{3}\right), 2.34(\mathrm{~m}, 1 \mathrm{H}, \mathrm{SH}), 2.46$ and $2.53(2 \mathrm{~m}, 4 \mathrm{H}$, $2 \mathrm{H}-1$ ', ' and 2H-2','), 3.34 (m, 1H, 1H-1' '), 3.42 (dd, $\left.J=14.4,8.4 \mathrm{~Hz}, 1 \mathrm{H}, 1 \mathrm{H}-1{ }^{\prime \prime}\right), 4.49$ (m, 1H, 1H2'’), 7.31 (d, $J=2.4 \mathrm{~Hz}, 1 \mathrm{H}, \mathrm{H}-2$ ') 7.36 (d, $J=8.8 \mathrm{~Hz}, 1 \mathrm{H}, \mathrm{H}-7$ '), 7.45 (d, $J=8.4 \mathrm{~Hz}, 1 \mathrm{H}, \mathrm{H}-3$ ), 7.67 (m, 1H, H-4), 7.69 (dd, $J=8.4,1.6 \mathrm{~Hz}, 1 \mathrm{H}, \mathrm{H}-6$ '), 7.73 (d, $J=1.6 \mathrm{~Hz}, 1 \mathrm{H}, \mathrm{H}-6), 8.41$ (d, $J=1.6 \mathrm{~Hz}$, 1H, H-4'), 9.44 (s, 1H, H-2a), 11.23 (d, $J=2.0 \mathrm{~Hz}, 1 \mathrm{H}, \mathrm{H}-1$ ').

3-[2-(2-Acetamido-5-carboxyphenyl)-1-(phenethylthio)ethyl]-1H-indole-5-carboxylic acid (8a). From 7a. HRMS: $\mathrm{M}+\mathrm{H}^{+}\left(\mathrm{C}_{28} \mathrm{H}_{27} \mathrm{~N}_{2} \mathrm{O}_{5} \mathrm{~S}\right)$ 503.1636; calculated 503.1640; Elemental analysis: found, \%: $\mathrm{N}$ 5.17, C 66.28, H 5.44; calculated for $3 \mathrm{C}_{28} \mathrm{H}_{26} \mathrm{~N}_{2} \mathrm{O}_{5} \mathrm{~S} \cdot \mathrm{H}_{2} \mathrm{O}$, \%: N 5.51. C 66.12, H 5.28.

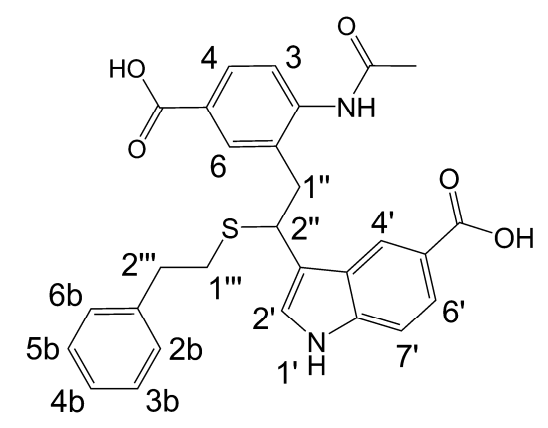

${ }^{1} \mathrm{H}-\mathrm{NMR}\left(270 \mathrm{MHz}, \mathrm{DMSO}-\mathrm{d}_{6}, 25^{\circ} \mathrm{C}\right): \delta 1.92\left(\mathrm{~s}, 3 \mathrm{H}, \mathrm{CH}_{3}\right), 2.55$ and 2.60 (2m, 4H, 2H-1',', 2H-2",'), 3.30-3.49 (m, 2H, 2H-1' '), 4.48 (m, 1H, H-2') 7.03 (XX' part of AA'XX'system, 2H, H-2b, H-6b), 7.08-7.22 (m, 1H, H-4b), 7.08-7.22 (AA' part of AA'XX'system, 2H, H-3b, H-5b), 7.32 (d, $J=2.3$ Hz, 1H, H-2'), 7.37 (d, $J=8.6$ Hz, 1H, H-7'), 7.47 (d, $J=8.3$ Hz, 1H, H-3), 7.69 (m, 2H, H-4, H-6'), 7.75 (d, $J=1.3 \mathrm{~Hz}, 1 \mathrm{H}, \mathrm{H}-6), 8.43$ (d, $J=1.3 \mathrm{~Hz}, 1 \mathrm{H}, \mathrm{H}-4$ '), 9.46 (br s, 1H, H-2a), 11.25 (d, $J=2.3$ $\left.\mathrm{Hz}, 1 \mathrm{H}, \mathrm{H}-1^{\prime}\right)$.

3-[2-(2-Acetamido-4-carboxyphenyl)-1-(phenethylthio)ethyl]-1H-indole-6-carboxylic acid (8b). From 7b. HRMS: $\mathrm{M}+\mathrm{H}^{+}\left(\mathrm{C}_{28} \mathrm{H}_{27} \mathrm{~N}_{2} \mathrm{O}_{5} \mathrm{~S}\right)$ 503.1656; calculated 503.1640; Elemental analysis: found, \%: $\mathrm{N}$ 5.30, C 65.24, H 5.17; calculated for $4 \mathrm{C}_{28} \mathrm{H}_{26} \mathrm{~N}_{2} \mathrm{O}_{5} \mathrm{~S} \cdot 3 \mathrm{H}_{2} \mathrm{O}, \%$ : N 5.43. C 65.16, H 5.37. 


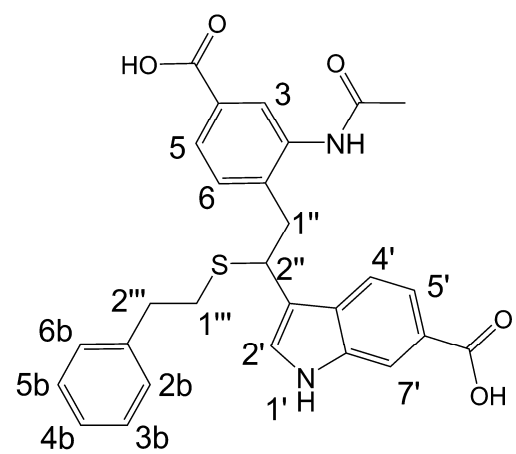

${ }^{1} \mathrm{H}-\mathrm{NMR}\left(270 \mathrm{MHz}, \mathrm{DMSO}-\mathrm{d}_{6}, 25^{\circ} \mathrm{C}\right): \delta 1.97$ (s, 3H, $\left.\mathrm{CH}_{3}\right), 2.54$ and 2.61 (2m, 4H, 2H-1',', 2H-2','), 3.31-3.51 (m, 2H, 2H-1' '), 4.50 (m, 1H, H-2') 7.04 (XX' part of AA'XX'system, 2H, H-2b, H-6b), 7.09-7.24 (m, 1H, 4b), 7.09-7.24 (AA' part of AA'XX'system, 2H, H-3b, H-5b), 7.09-7.24 (m, 1H, H6), $7.42(\mathrm{~d}, J=2.6 \mathrm{~Hz}, 1 \mathrm{H}, \mathrm{H}-2$ ') 7.51 (dd, $J=7.9,2.0 \mathrm{~Hz}, 1 \mathrm{H}, \mathrm{H}-5), 7.57(\mathrm{dd}, J=8.3,1.6 \mathrm{~Hz}, 1 \mathrm{H}, \mathrm{H}-$ 5'), 7.73 (d, $\left.J=8.3 \mathrm{~Hz}, 1 \mathrm{H}, \mathrm{H}-4^{\prime}\right), 7.84$ (d, $\left.J=2.0 \mathrm{~Hz}, 1 \mathrm{H}, \mathrm{H}-3\right), 7.96$ (d, $J=1.6 \mathrm{~Hz}, 1 \mathrm{H}, \mathrm{H}-7$ '), 9.48 (br s, 1H, H-2a), 11.27 (d, $J=2.3 \mathrm{~Hz}, 1 \mathrm{H}, \mathrm{H}-1^{\prime}$ ).

3-\{2-[2-Acetamido-5-(diethylcarbamoyl)phenyl]-1-(phenethylthio)ethyl\}-N,N-diethyl-1H-indole-5carboxamide (8c). From 7c. HRMS: $\mathrm{M}+\mathrm{H}^{+}\left(\mathrm{C}_{36} \mathrm{H}_{45} \mathrm{~N}_{5} \mathrm{O}_{3} \mathrm{~S}\right)$ 613.3205; calculated 613.3212; Elemental analysis: found, \%: $\mathrm{N} 8.63, \mathrm{C} 69.01, \mathrm{H}$ 7.03; calculated for $6 \mathrm{C}_{36} \mathrm{H}_{44} \mathrm{~N}_{5} \mathrm{O}_{3} \mathrm{~S} \cdot \mathrm{CF}_{3} \mathrm{COOH} \mathrm{H} \mathrm{H}_{2} \mathrm{O}, \%$ : $\mathrm{N} 8.83$. C 68.74, H 7.07.

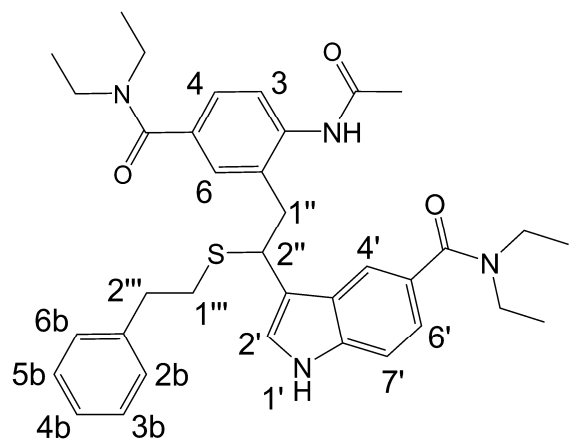

${ }^{1} \mathrm{H}-\mathrm{NMR}\left(400 \mathrm{MHz}, \mathrm{DMSO}-\mathrm{d}_{6}, 25^{\circ} \mathrm{C}\right): \delta 0.74$ and $1.08(2 \mathrm{~m}, 12 \mathrm{H}, 4 \mathrm{CH} 3), 1.99\left(\mathrm{~s}, 3 \mathrm{H}, \mathrm{COCH}_{3}\right), 2.51-$ 2.66 (m, 4H, 2H-1',', 2H-2'"'), 3.24-3.44 (m, 10H, 4CH ${ }_{2}$ NEt, 2H-1'”), 4.46 (dd, J=8.8, 6.4 Hz, 1H, H-2' '), 6.89 (d, $J=2.0 \mathrm{~Hz}, 1 \mathrm{H}, \mathrm{H}-6), 7.02-7.06$ (XX' part of AA'XX'system, 2H, H-2b, H-6b), 7.027.06 (m, 2H, H-4, H-6'), 7.09-7.13 (m, 1H, H-4b), 7.16-7.20 (AA' part of AA'XX'system, 2H, H-3b, H-5b), 7.23 (d, $J=2.4$ Hz, 1H, H-2'), 7.31 (d, $J=8.0$ Hz, 1H, H-3), 7.32 (d, $J=8.4$ Hz, 1H, H-7'), 7.68 (m, 1H, H-4'), 9.45 (br s, 1H, H-2a), 11.06 (d, $J=2.4$ Hz, 1H, H-1').

\section{References and Notes}

1. Fruechtel, J. S.; Jung, G. Organic chemistry on solid supports. Angew. Chem. Int. Ed. Engl. 1996, 35, 17-42.

2. Brown, R. C. D. Recent developments in solid-phase organic synthesis. J. Chem. Soc., Perkin Trans. 1 1998, 3293-3320.

3. Crowley, J. I.; Rapoport, H. Solid- Phase Organic Synthesis: Novelty or Fundamental Concept? Acc. Chem. Res. 1976, 9, 135-144. 
4. Mutulis, F. unpublished data.

5. Keller, K., Polymeric Indoles. Chem. Ber. 1913, 46, 726-733.

6. Wu, Y-H.; Lobeck, W. G., Jr.; Ryan, R. P. Diuretics. 1. 1-Imidoyl-2-(2- and 3-indolyl)indolines. J. Med. Chem. 1972, 15, 529-534.

7. Bergman, J.; Egestad, B.; Eklund, N. The reaction of indigo with hydrazine. Tetrahedron Lett. 1978, 34, 3147-3150.

8. Banerji, J.; Saha, R.; Manna, A. P.; Shoolery, J. N.; Prange, T.; Pascard, C. Electrophilic substitution of indoles: Part IV - Addition and annulation reactions with mesityl oxide. Indian J. Chem. 1982, 21B, 83-87.

9. Napolitano, A.; Corradini, M. G.; Prota, G. A reinvestigation of the structure of melanochrome. Tetr. Lett. 1985, 26, 2805-2808.

10. Somei, M.; Hayashi, H.; Ohmoto, S. Reduction of indigo: simple syntheses of 3-acetoxy-, 1acetyl-2,3-dihydro-, 3-acetoxy-3'-acetyl-, 3-acetoxy-1,3'-diacetyl-2,2'-bisindoles, and 2,2'bisindole. Heterocycles 1997, 44, 169-176.

11. Smith, G. F. The dimerization and trimerization of indole. Chem. Ind. 1954, 1451-1452.

12. Ishii, H.; Murakami, K.; Murakami, Y.; Hosoya, K. Fischer indolization and its related compounds. XI. New indole trimer. Chem. Pharm. Bull. 1977, 25, 3122-3124.

13. Ishii, H.; Murakami, K.; Furuse, T.; Hosoya, K. Abnormal Fischer indolization and its related compounds. XII. Synthesis of 3,6'-biindole. Chem. Pharm. Bull 1979, 27, 346-350.

14. Bocchi, V.; Palla, G. Synthesis and characterization of new indole trimers and tetramers. Tetrahedron 1986, 42, 5019-5024.

15. Ishii, H.; Sakurada, E.; Murakami, K.; Takase, S.; Tanaka, H. Polymerization of indole. Part 3. Two indolylquinolines, an indole tetramer, and the dihydro derivative of the indole dimer. $J$. Chem. Soc. Perkin Trans. I 1988, 2387-2395.

16. Mackintosh, J. G.; Mount, A. R.; Reed, D. Characterization of the unsymmetrical trimer of indole-5-carboxylic acid by proton NMR spectroscopy. Magn. Reson. Chem. 1994, 32, 559-561.

17. Manini, P.; d'Ischia, M.; Milosa, M.; Prota, G. Acid-Promoted Competing Pathways in the Oxidative Polymerization of 5,6-Dihydroxyindoles and Related Compounds: Straightforward Cyclotrimerization Routes to Diindolocarbazole Derivatives. J. Org. Chem. 1998, 63, 7002-7008.

18. Sanders, J. K. M.; Hunter, B. K. Modern NMR Spectroscopy; A Guide for Chemists, 2nd Ed. Oxford University Press: Oxford, U.K., 1993.

19. Bax, A.; Freeman, R.; Gareth, M. Correlation of proton chemical shifts by two-dimensional Fourier transform NMR. J. Magn. Reson 1981, 42, 164-168.

20. Breitmaier, E.; Voelter, W. Carbon-13 NMR Spectroscopy; High-resolution Methods and Applications in Organic Chemistry and Biochemistry, 3rd Ed. VCH Verlagsgesellsschaft: Weinhem, 1987.

21. Reynolds, W. F.; McLean, S.; Perpick-Dumont, M.; Enriquez, R. G. Carbon-13-proton shift correlation with full proton-proton decoupling. II. Further significant improvements in resolution and sensitivity. Magn. Reson. Chem. 1988, 26, 1068-1074.

22. Bax, A. Structure determination and spectral assignment by pulsed polarization transfer via longrange proton-carbon-13 couplings. J. Magn. Res. 1984, 57, 314-318. 
23. King, D. S.; Fields, C. G.; Fields, G. B. A cleavage method which minimizes side reactions following Fmoc solid phase peptide synthesis. Int. J. Pept. Protein Res. 1990, 36, 255-266.

24. Sundberg, R. J. The Chemistry of Indoles. Academic Press: New York, 1970.

25. Igolen, J.; Kolb, A. 4(and 5)-Substituted indoles prepared from 5-bromoindole. C. R. Acad. Sci., Ser. C: Chim. 1969, 269, 54-56.

26. Firouzabadi, H.; Iranpoor, N.; Jafarpour, M.; Ghaderi, A. Silica gel catalyzed highly selective C-S bond formation via Michael addition of thiols to $\alpha, \beta$-unsaturated ketones under solvent-free conditions. J. Mol. Catal. A: Chem. 2006, 249, 98-102.

Sample Availability: Samples of the compounds are available from the authors upon request.

(C) 2008 by the authors; licensee Molecular Diversity Preservation International, Basel, Switzerland. This article is an open-access article distributed under the terms and conditions of the Creative Commons Attribution license (http://creativecommons.org/licenses/by/3.0/). 